\title{
Planetary period oscillations in Saturn's magnetosphere: comments on the relation between post-equinox periods determined from magnetic field and SKR emission data
}

\author{
S. W. H. Cowley and G. Provan \\ Department of Physics and Astronomy, University of Leicester, Leicester LE1 7RH, UK \\ Correspondence to: S. W. H. Cowley (swhc1@le.ac.uk) \\ Received: 15 April 2015 - Revised: 22 June 2015 - Accepted: 1 July 2015 - Published: 24 July 2015
}

\begin{abstract}
We discuss the properties of Saturn planetary period oscillations (PPOs) deduced from analysis of Saturn kilometric radiation (SKR) modulations by Fischer et al. (2014), and from prior analysis of magnetic field oscillations data by Andrews et al. (2012) and Provan et al. (2013), with emphasis on the post-equinox interval from early 2010 to early 2013. Fischer et al. (2014) characterize this interval as showing single phase-locked periods in the northern and southern SKR modulations observed in polarizationseparated data, while the magnetic data generally show the presence of separated dual periods, northern remaining shorter than southern. We show that the single SKR period corresponds to the southern magnetic period early in 2010, segues into the northern period in late 2010, and returns to the southern period in mid-2012, approximately in line with changes in the dominant magnetic oscillation. An exception occurs in mid-February to late August 2011 when two periods are again discerned in SKR data, in good agreement with the ongoing dual periods in the magnetic data. Fischer et al. (2014) discuss this change in terms of a large jump in the southern SKR period related to the Great White Spot storm, which the magnetic data show is primarily due instead to a reappearance in the SKR data of the ongoing southern modulation in a transitory interval of resumed southern dominance. In the earlier interval from early April 2010 to mid-February 2011 when Fischer et al. (2014) deduce single phase-locked periods, we show unequivocal evidence in the magnetic data for the presence of separated dual oscillations of approximately equal amplitude. We suggest that the apparent single SKR periods result from a previously reported phenomenon in which modulations associated with one hemisphere appear in polarization-separated data associated with the other. In the following interval, mid-August 2011 to early
\end{abstract}

April 2012, when Fischer et al. (2014) again report phaselocked northern and southern oscillations, no ongoing southern oscillation of separate period is discerned in the magnetic data. However, the magnetic amplitude data show that if a phase-locked southern oscillation is indeed present, its amplitude must be less than $\sim 5-10 \%$ of the northern oscillation.

Keywords. Magnetospheric physics (planetary magnetospheres)

\section{Introduction}

The phenomenon of planetary period oscillations (PPOs) has formed an important topic in studies of Saturn's magnetosphere during the Cassini era. Observations have shown that two seasonally changing oscillations are present with slightly different periods, one associated with the northern hemisphere and the other with the southern (Galopeau and Lecacheux, 2000; Kurth et al., 2008; Gurnett et al., 2009). The physical picture emerging is one in which rotating current systems are driven from the two ionospheres, most probably through coupling to rotating twin-vortex flows in the polar thermosphere (Smith, 2010; Jia et al., 2012; Southwood and Cowley, 2014). The planetary field communicates this force to the magnetospheric plasma via a current system that rotates wave-like through the sub-corotating plasma, thus leading to rotating magnetic field and plasma perturbations throughout the magnetosphere (e.g. Carbary et al., 2007; Andrews et al., 2008, 2010a; Burch et al., 2009). The force from one hemisphere may also be communicated to the other if the associated field-aligned currents (FACs) extend 
into an inter-hemispheric system (Southwood and Kivelson, 2007). PPO-modulation of the FACs associated with plasma sub-corotation and the solar wind interaction also leads to modulated auroral electron acceleration in regions of upward FAC (Nichols et al., 2008, 2010a, b; Southwood and Kivelson, 2009; Badman et al., 2012; Hunt et al., 2014), together with associated modulated radio emissions at kilometre wavelengths generated via the cyclotron maser instability (Zarka, 1998; Lamy et al., 2010, 2011). The latter emission is termed Saturn kilometric radiation (SKR), through which the PPO phenomenon was first observed in Voyager data (Warwick et al., 1981, 1982; Gurnett et al., 1981).

To date, the phases and periods of the PPOs have been tracked near-continuously during the Cassini mission using both SKR data (e.g. Kurth et al., 2008; Lamy, 2011; Gurnett et al., 2011) and magnetic field data obtained when the spacecraft is located inside the magnetosphere (e.g. Andrews et al., 2012; Provan et al., 2013, 2014). These analyses are in good general agreement that the two periods were well-separated at $\sim 10.6 \mathrm{~h}$ for the northern period and $\sim 10.8 \mathrm{~h}$ for the southern period during southern summer conditions early in the Cassini mission, and that they converged towards $\sim 10.7 \mathrm{~h}$ over a $\sim 1$ Earth-year interval spanning Saturn equinox in mid-August 2009. Since both magnetic perturbations and SKR modulations are believed to be associated with the same rotating current systems, the rotation periods are expected in principle to be identical. However, somewhat different results have been reported for the post-equinox interval (Provan et al., 2013, 2014; Fischer et al., 2014), which it is the purpose of this paper to discuss.

A potentially significant issue arises in the determination of phases and periods from SKR modulation data from the "dual modulations" that are sometimes observed in the emission apparently from a given hemisphere. The conically beamed SKR sources in one hemisphere generally illuminate the region to $\sim 20^{\circ}$ below the equator in the other (Lamy et al., 2008a; Kimura et al., 2013), with the exception of a nearplanet shadow zone extending $\sim 4 R_{\mathrm{S}}$ from the planet in the equatorial plane (Lamy et al., 2008b) $\left(R_{\mathrm{S}}\right.$ is Saturn's 1 bar equatorial radius equal to $60268 \mathrm{~km}$ ). Consequently, nearequatorial SKR observations outside this radius, corresponding to a significant proportion of all Cassini observations, will generally observe modulated emission from both hemispheres. However, as first shown by Lamy (2011), these can be distinguished by separating the emissions with respect to their sense of circular polarization, emissions from the north and south being predominantly right-hand (RH) and lefthand $(\mathrm{LH})$ polarized, respectively, corresponding to the extraordinary mode. It is notable, however, that during some intervals, modulations associated with one hemisphere are also observed in the polarization-separated data from the other hemisphere so that dual modulations are present (e.g. Fig. 1 of Lamy, 2011). The origin of this effect is at present uncertain: it is not clear whether it is a real physical phenomenon due, e.g. to unstable auroral electron distributions propagat- ing along the field from one hemisphere to the other or to inter-hemispheric currents as mentioned above, or whether it is an effect of the polarization-separation technique. It is notable, however, that the effect appears to be more prevalent in near-equatorial data, corresponding to the majority of that discussed here, rather than in high-latitude data when the spacecraft is largely located in the shadow zone of emissions from the opposite hemisphere.

Under most circumstances the resulting ambiguity in the hemispheric origin of the SKR modulations is readily resolved from consideration of the continuity of the data in both polarization channels, as well as comparison with data from adjacent intervals at higher latitudes. This is certainly true of the pre-equinox data studied by Lamy (2011). However, the dual modulation effect gives rise to the potential for confusion post-equinox, when the northern and southern periods are closer together, with a difference in period of $\sim 0.05 \mathrm{~h}$ compared with $\sim 0.2 \mathrm{~h}$ during southern summer, and where the two modulations are found in magnetic field data to undergo large simultaneous abrupt (revolutionto-revolution; rev-to-rev) changes in relative amplitude from southern to northern dominance, and vice versa, at intervals of 100-200 days (Provan et al., 2013, 2014). If in a particular interval the "secondary" modulation from a particular hemisphere (i.e. a particular sense of circular polarization) at the period of the opposite hemisphere is of lesser amplitude than that of the "primary", then following the principal peaks of the emission in time, as, e.g. in Fig. 5 of Fischer et al. (2014), will reveal the primary phase and hence period associated with that hemisphere. However, if we suppose that the relative amplitude of the modulations changes significantly, such that the amplitude of the secondary should exceed that of the primary, then the same procedure will reveal the period associated with the opposite hemisphere, regardless of whether or not a lesser primary modulation is still present. Not only this, but the phase of the secondary emission will correspond exactly (within observation uncertainties) to that in the opposite hemisphere, i.e. it will appear that the modulations from the two hemispheres are "phaselocked" at a common period. If changes in the relative amplitudes of the two modulations then result in an abrupt switch between these two conditions in the emissions from a given hemisphere (i.e. circular polarization state), the modulations from that hemisphere will appear to undergo an abrupt switch to or from the phase-locked period of the opposite hemisphere. As we will suggest below, just such conclusions have recently been drawn by Fischer et al. (2014) studying SKR modulations in the post-equinox interval, while analyses of the magnetic field oscillations clearly show two oscillations with separated periods generally to be present. 


\section{Comparison of PPO periods determined from SKR and magnetic field data}

Figure 1 provides an overview of the PPO periods determined from Cassini SKR modulation and magnetic field oscillation data spanning the interval from the beginning of 2004 to mid-2013. Saturn vernal equinox in mid-August 2009 is marked by the vertical arrow. The coloured spectrogram is taken from Fig. 1 of Fischer et al. (2014), here inverted to show the period vs. time in days, where throughout this paper $t=0$ corresponds to 00:00 UT on 1 January 2004. This shows the peak-to-peak modulation power of the complete SKR emission integrated over $80-500 \mathrm{kHz}$ (i.e. not separated by circular polarization), thus in principle exhibiting modulations associated with both hemispheres. Analysis details are given by Gurnett et al. $(2009,2011)$, but we note here that the powers were derived using a 240-day data window shifted in 30-day steps. The over-plotted red and blue lines show the southern and northern periods, respectively, derived from magnetic field data over the same interval by Andrews et al. (2012) and Provan et al. (2013, 2014). These periods were determined from rev-to-rev phase measurements of the PPO magnetic oscillations observed within the quasi-dipolar magnetospheric "core" region (dipole $L \leq 12$ ) on few-day near-equatorial Cassini periapsis passes where the northern and southern oscillations combine to produce beats, augmented with polar phase data obtained on highly inclined orbits where the oscillations are found to be purely characteristic of the hemisphere concerned (Andrews et al., 2010b, 2012). The nature of the orbits, and hence of the magnetic phase data, is indicated by the coloured bar at the top of the panel, where blue indicates near-equatorial orbits and green highly inclined, lettered sequentially from the start of the inorbit mission (mid-2004). The periods were determined from running $~ 200$-day segments of phase data evaluated every $\sim 10$ days for intervals A-E1 (Andrews et al., 2012), thus similar to the intervals employed in the SKR analysis. Beyond E1, however, a sequence of abrupt (rev-to-rev) changes in the magnetic PPO properties were observed at $\sim 100-200$ day intervals shown by the white dotted lines, characterized principally by simultaneous changes in the amplitudes of the two oscillations, together with small changes in the oscillation periods and phases. Only single linear fits to the phase data yielding single values for the northern and southern periods have been determined in these short intervals (Provan et al., 2013, 2014). The vertical dashed lines in Fig. 1 also show the interval of the Saturn Great White Spot (GWS) storm, a central theme of the discussion by Fischer et al. (2014), specifically the interval of storm-associated lightning strokes observed in radio data, from initiation on 5 December 2010 to complete cessation on 28 August 2011.

Prior to equinox two clearly separated periods were present in both data sets, the longer $\sim 10.8 \mathrm{~h}$ corresponding to the southern oscillation, and the shorter $\sim 10.6 \mathrm{~h}$ to the northern, thus separated by $\sim 0.2 \mathrm{~h}$ (i.e. $\sim 2 \%$ ). The agree- ment between SKR and magnetic determinations is generally excellent (Andrews et al., 2012), with the exception of some minor deviations in intervals of weak northern magnetic oscillations indicated by the blue and white dashed lines. During the $\sim 1$-year interval across the equinox, however, the two oscillations converged towards a common period of $\sim 10.67 \mathrm{~h}$, and remained in this vicinity, separated by $\sim 0.05 \mathrm{~h}$, until the end of the interval shown. Fischer et al. (2014) interpret the SKR data as indicating a crossing of the two periods post-equinox until early 2010 , following which there is generally only one SKR period until early 2013, shown in Fig. 1 by the black dashed line. An exception occurs in an interval starting mid-February 2011 (E2 in the top bar) in which separated periods are again apparent in the SKR data, which the authors associate with the GWS. However, the magnetic data presented by Andrews et al. (2012) and Provan et al. (2013, 2014), and referenced but not discussed by Fischer et al. (2014), shows northern and southern periods to be present essentially throughout the post-equinox interval, separated by $\sim 0.05 \mathrm{~h}$, with the southern period remaining longer than the northern. An exception in this case occurs during the second half of 2011 to early 2012 (interval E3) when no southern oscillations were detected in the magnetic data, such that no southern period is then shown in the figure. Comparison with the magnetic periods shows that in E1 the single period identified by Fischer et al. (2014) corresponds approximately to the southern magnetic period in early-to-mid-2010 but segues into the northern magnetic period in late 2010 and early 2011 , remains at the northern period throughout 2011 and into 2012 (E2 and E3), and then segues back to the southern magnetic period in mid-2012 (interval E4). This variation approximately mirrors the behaviour of the relative amplitudes of the northern and southern oscillations observed in the equatorial magnetic data by Provan et al. (2013, 2014). The amplitudes were modestly southern-dominant in the initial part of E1 but became modestly northern-dominant towards its end, and then became strongly northern-dominant in E3 before relaxing again from less strongly northern-dominant to weakly southern-dominant across E4, F1 and F2 (see Sect. 3). The exception is interval E2, where, as discussed below, the single period indicated by the black dashed line in Fig. 1 continues to correspond to the northern period, while the magnetic data show the resumption of strong southern dominance $(k \approx 0.3)$, in line with the second SKR period that is evident in Fig. 1. Although the post-equinox SKR and magnetic periods are thus often seen to be in good agreement in Fig. 1, it is notable that there is no obvious counterpart in the SKR data of the northern magnetic period in the post-equinox interval from late 2009 to late 2010 (i.e. throughout much of E1), or of the southern magnetic period between late 2010 and early 2011 (i.e. the end of E1), or of the northern magnetic period late in 2012 in E4, despite the magnetic oscillations providing clear evidence of their existence. 


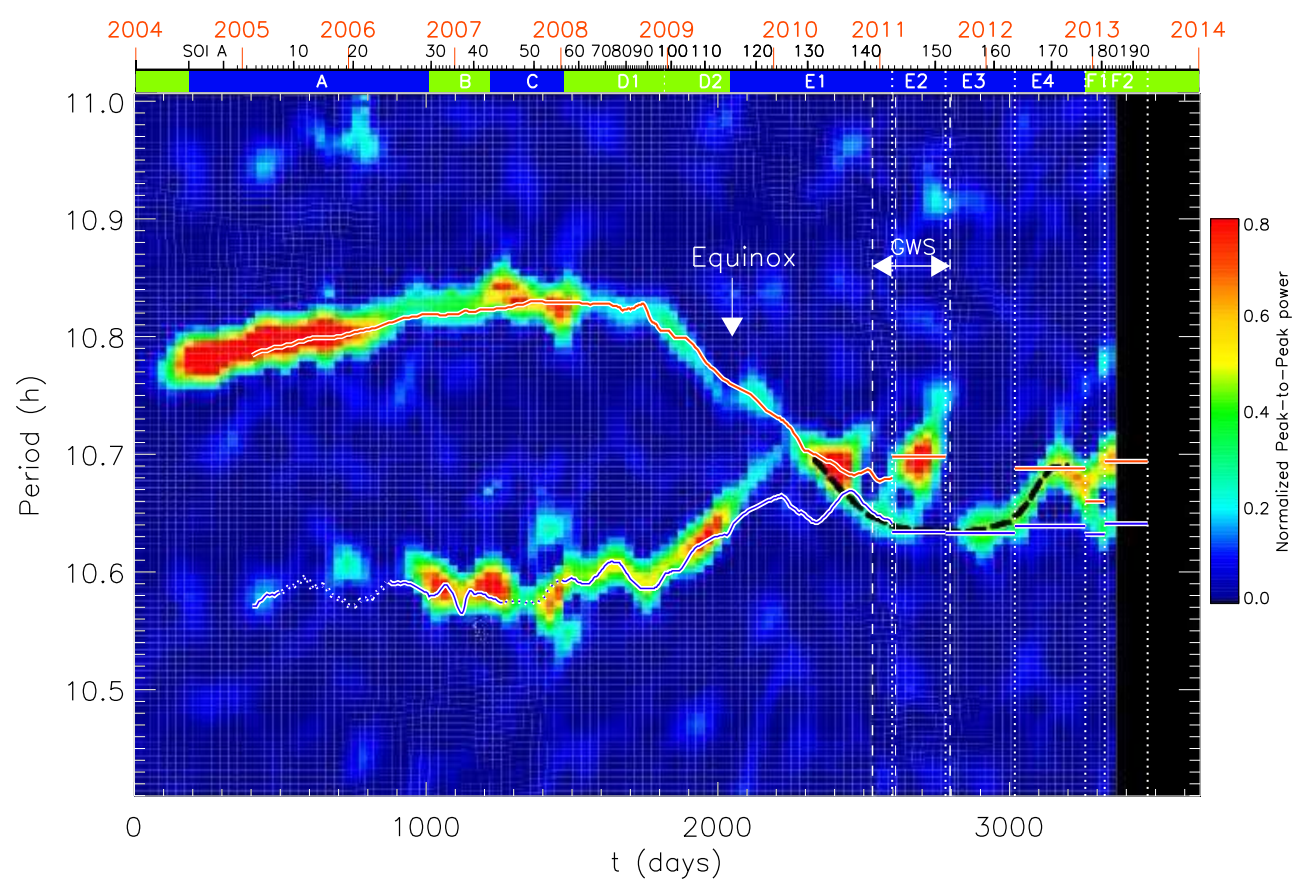

Figure 1. Spectrogram of the normalized peak-to-peak power of SKR modulations taken from Fig. 1 of Fischer et al. (2014), shown here as modulation period vs. time in days since 00:00 UT on 1 January 2004, over the interval from the beginning of 2004 to mid-2013. At the top we show the start of each year, the Cassini rev numbers defined from apoapsis to apoapsis and plotted at periapsis, and a coloured bar which indicates the nature of the Cassini orbit either near-equatorial (blue) or highly inclined (green), lettered from A to F, which determines the nature of the data employed to undertake the magnetic field PPO analysis. Over-plotted are the PPO periods determined from the magnetic field analysis of Andrews et al. (2012) and Provan et al. (2013, 2014), red for the southern period and blue for the northern (shown dashed when the northern oscillations were not prominent in these data). The vertical white dotted lines indicate the times of six abrupt transitions in the PPO properties observed in the magnetic field data post-equinox (indicated by the white vertical arrow), defining intervals E2-F2 (as in Provan et al., 2013, 2014). No southern magnetic field oscillation was discerned in E3 such that no southern period is shown in that interval. The white vertical dashed lines indicate the interval of the GWS, shown as in Fischer et al. (2014) from the start of storm-associated lightning activity to its complete cessation (5 December 2010 to 28 August 2011).

Results using polarization-separated SKR data are also shown by Fischer et al. (2014) in their Figs. 5-7, where emission intensity modulations were tracked by eye, augmented by directional statistics analysis, to yield phases vs. time and hence periods. As above, they report corresponding modulations in both polarization channels throughout the interval indicated by the black dashed line in Fig. 1 indicative of a single phase-locked period, with the exception of interval E2, when dual modulations become evident. Figure 2 compares these periods with those derived by Andrews et al. (2012) and Provan et al. (2013, 2014) over the interval from the beginning of 2010 to mid-2012 (later E1 to early E4), where the circles joined by dotted lines show the SKR periods (Fischer et al., 2014, Fig. 6) and the solid lines the magnetic periods, where red data show the southern period and blue the northern. The solid squares during E1 also show magnetic periods derived by Provan et al. (2013) using an alternative multi-parameter fit which employs 150-day intervals of phase data separated by 50 days. Figure 3 also shows the phase difference (modulo $360^{\circ}$ ) between the two oscillations over the same interval, northern phase minus southern phase, where the green line shows the SKR data (Fischer et al., 2014, Fig. 7) and the purple line the magnetic. No magnetic data are shown in E3 since southern oscillations were not discerned in this interval as indicated above, but resume in E4 with an arbitrarily assigned modulo $360^{\circ}$ value.

At the beginning of the interval in Figs. 2 and 3, the SKR modulations indicate separate northern and southern periods, with the northern longer than the southern, but become essentially the same near $t \approx 2300$ days (mid-April 2010) and remain so until the beginning of E2 near $t \approx 2600$ days (mid-February 2011). The SKR phase difference in Fig. 3 also varies about zero during the latter interval, such that the phases of the northern and southern (polarization-separated) oscillations are approximately equal (modulo $360^{\circ}$ ), i.e. phase-locked, as well. The two SKR periods then separate in E2, the southern being longer than the northern such that the phase difference in Fig. 3 increases by three cycles over the interval, before coalescing again at the beginning of E3 just prior to $t \approx 2800$ days (late August 2011) to a common value similar to that at the end of $\mathrm{E} 1$ and the northern period in E2. The phase difference in E3 is again near zero (modulo 


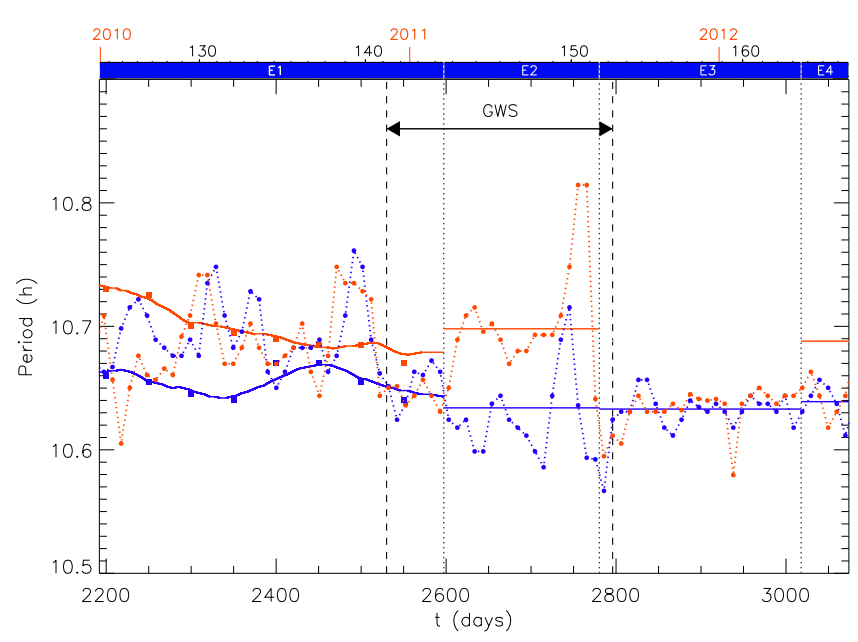

Figure 2. Plot of the PPO periods vs. time from the start of 2010 to mid-2012. Values derived from SKR modulations are shown by circles joined by dotted lines (from Fig. 6 of Fischer et al., 2014), while values derived from magnetic field phase data are shown by solid lines (from Andrews et al., 2012 and Provan et al., 2014) and squares (Provan et al., 2013). Blue data correspond to the northern oscillations and red to the southern. Year boundaries, rev numbers, and interval identifiers are shown at the top of the plot as in Fig. 1, together with vertical dashed and dotted lines as also in Fig. 1.

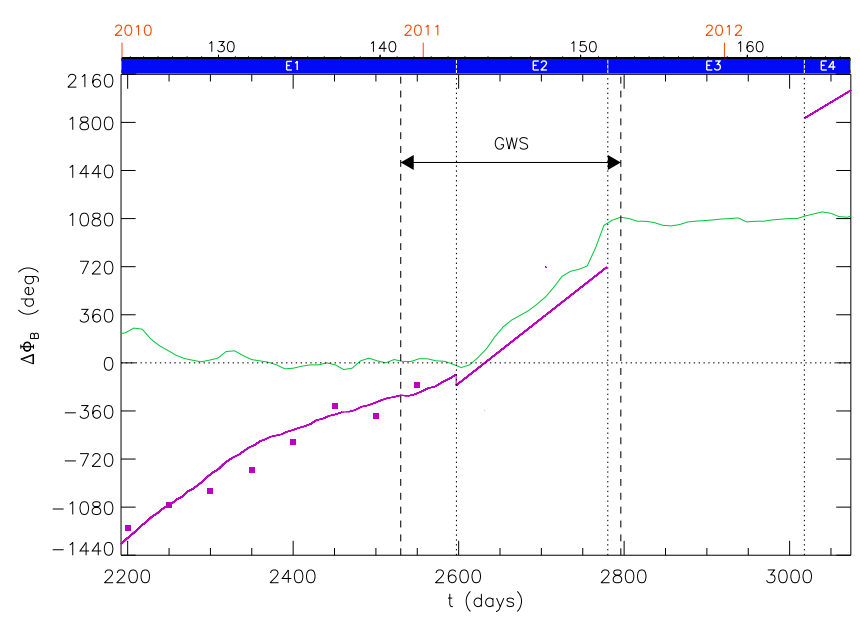

Figure 3. Phase difference (modulo $360^{\circ}$ ) between the northern and southern PPO oscillations (northern minus southern), $\Delta \Phi_{B}$, plotted vs. time between the start of 2010 and mid-2012. The green line was derived from SKR modulation data (from Fig. 7 of Fischer et al., 2014), corresponding to the circles joined by dotted lines in Fig. 2, while the purple line and squares were derived from magnetic field data by Andrews et al. (2012) and Provan et al. (2013, 2014), corresponding to the solid lines and squares in Fig. 2. No magnetic data are shown in E3 since no southern oscillation was discerned in these data, such that the phase in E4 is shown starting at an essentially arbitrary modulo $360^{\circ}$ value. Year boundaries, rev numbers, and interval identifiers are shown at the top of the plot as in Fig. 2, together with vertical dashed and dotted lines as also in Fig. 2. $360^{\circ}$ ), i.e. the two modulations are again phase-locked. Comparison with the magnetic periods in Fig. 2 shows little correspondence at the start of the interval early in 2010, where the SKR periods are apparently "reversed" (northern longer than southern) compared with the magnetic, but the phase-locked SKR periods in E1 are seen to correspond to the southern magnetic period between $t \approx 2300$ and $\sim 2450$ days (late April to mid-September 2010), and to the northern magnetic period between $\sim 2500$ and $\sim 2600$ days (late October 2010 to mid-February 2011), as indicated above. The phase difference of the magnetic oscillations in Fig. 3 undergoes $\sim$ two clear beat cycles during the interval in E1 in which the SKR modulations remain phase-locked. Overall, the northern and southern SKR and magnetic periods then agree well in E2, and show closely similar phase gradients in Fig. 3. Following this, in E3, the resumed phase-locked SKR modulations have a closely similar period to the single northern magnetic period, while in E4 the similarly phase-locked SKR modulations have a closely similar period to the northern magnetic period and not the southern. As discussed in Sect. 1, we here suggest that the phase-locked single periods deduced from the SKR data, but not the magnetic data, are likely due to the phenomenon first noted by Lamy (2011), in which the SKR modulation associated with a dominant hemisphere also appears in the (polarization-separated) data for the other.

The different perceptions to which these analyses give rise, however, can lead to significant consequences. In discussing the SKR periods in relation to the GWS in their Sect. 2, Fischer et al. (2014) characterize the interval in Fig. 2 as showing a "large jump discontinuity" of the southern modulations to a longer period in E2 compared with the southern phaselocked periods they deduce at the end of E1 and in E3, the magnitude of the jump being $\sim 0.05 \mathrm{~h}$. They suggest that this "unique feature" is associated with the presence of the GWS, with which it is approximately contemporaneous. However, the magnetic results instead suggest that these effects result primarily from changes in the relative amplitude of the northern and southern oscillations, from northern-dominant at the end of E1, to southern dominant in E2, and then back to northern-dominant in E3, thus leading to the temporary reappearance of southern modulations in the SKR data, with the oscillation periods, to a first approximation, ongoing. The magnetic data do indicate an increase in the southern period across the E1/E2 boundary, but by $\sim 0.02 \mathrm{~h}$, less than half that discussed by Fischer et al. (2014), while no conclusions can be drawn at all concerning changes in the southern period at the following E2/E3 boundary since no southern oscillations were observed in E3. We note, however, that when southern oscillations resume in interval E4 the period is again similar to that determined in E2 (and E1), within $\sim 0.01 \mathrm{~h}$. The variation in the northern oscillation periods between the end of E1 and E4 is of similar magnitude, less than $\sim 0.01 \mathrm{~h}$.

In following sections we further address issues related to the discrepancies noted above in the PPO periods derived from magnetic and SKR data, a topic on which Fischer et 
al. (2014) did not comment. Specifically, in Sect. 3 we review the magnetic PPO data, focussing on the evidence for separated dual periods in E1 when single phase-locked northern and southern periods were deduced by Fischer et al. (2014). In Sect. 4 we also quantify and strongly limit the possible amplitude of a phase-locked southern oscillation in E3.

\section{Post-equinox magnetic oscillations and relation to SKR modulations in interval E1}

The amplitudes and phases of the oscillations in the three spherical polar field components $i$ (referenced to the planetary spin and magnetic axes), observed during each few-day periapsis pass through the core magnetosphere, are obtained by fitting the data to the azimuthally rotating function

$B_{i}(\varphi, t)=B_{0 i} \cos \left(\Phi_{\mathrm{g}}(t)-\varphi-\psi_{i}\right)$,

where $\Phi_{\mathrm{g}}(t)$ is a guide phase corresponding to a fixed period $\tau_{\mathrm{g}}$ close to that of the oscillations $\left(\Phi_{\mathrm{g}}(t)=\left(360 t / \tau_{\mathrm{g}}\right) \mathrm{deg}\right)$, and $\varphi$ is azimuth measured from noon increasing in the sense of planetary rotation, thus determining $B_{0 i}$ and $\psi_{i}$ for each component $i=(r, \theta, \varphi)$ on each pass. The time variations of the amplitudes and phases are then given by the rev-to-rev data, with total phase

$\Phi_{i}(t)=\Phi_{\mathrm{g}}(t)-\psi_{i}(t)$.

The observed oscillations are taken to consist of the sum of northern $(\mathrm{N})$ and southern $(\mathrm{S})$ contributions,

$$
\begin{aligned}
B_{0 i}(t) \cos \left(\Phi_{i}(t)-\varphi\right) & =B_{0 \mathrm{~N} i} \cos \left(\Phi_{\mathrm{N}}(t)-\varphi-\gamma_{i \mathrm{~N}}\right) \\
& +B_{0 \mathrm{~S} i} \cos \left(\Phi_{\mathrm{S}}(t)-\varphi-\gamma_{i \mathrm{~S}}\right),
\end{aligned}
$$

where $B_{0 \mathrm{~N}, \mathrm{~S} i}$ are the amplitudes of the two oscillations with north / south ratio $k=B_{0 \mathrm{~N} i} / B_{0 \mathrm{~S} i}$ assumed the same for each field component, $\Phi_{\mathrm{N}, \mathrm{S}}(t)$ are the magnetic phases with corresponding periods $\tau_{\mathrm{N}, \mathrm{S}}=360 /\left(\mathrm{d} \Phi_{\mathrm{N}, \mathrm{S}} / \mathrm{d} t\right)$ if the phases are expressed in degrees, and the constant angles $\gamma_{i \mathrm{~N}, \mathrm{~S}}$ describe the relative phases of the three field components of the two systems, where by definition we take $\gamma_{r \mathrm{~N}, \mathrm{~S}} \equiv 0^{\circ}$ such that $\Phi_{\mathrm{N}, \mathrm{S}}(t)$ corresponds explicitly to the phases of the $r$ components. Observations then show that $\gamma_{\varphi \mathrm{N}, \mathrm{S}}=90^{\circ}$ for both systems, such that the $\varphi$ components are in lagging quadrature with $r$ in the equatorial region, forming a rotating quasiuniform perturbation field, while $\gamma_{\theta \mathrm{S}}=0^{\circ}$ and $\gamma_{\theta \mathrm{N}}=180^{\circ}$, such that the $\theta$ components are in phase with $r$ for the southern system, but in anti-phase with $r$ for the northern. The phase $\Phi_{i}(t)$ and the amplitude $B_{0 i}(t)$ of the observed combined oscillations are then modulated at the beat period of the two oscillations $\tau_{B}(t)=360 /\left(\mathrm{d} \Delta \Phi_{B} / \mathrm{d} t\right)$, where $\Delta \Phi_{B}$ is the beat phase

$\Delta \Phi_{B}(t)=\Phi_{\mathrm{N}}(t)-\Phi_{\mathrm{S}}(t)$.

The form of the modulations depends on both $\Delta \Phi_{B}$ and $k$ (Andrews et al., 2012; Provan et al., 2013), such that in general both northern and southern phases can be determined by fits to the modulated phase data, together with the amplitude ratio. The individual component amplitudes can then be found by fits to the observed amplitude data. Practically, the limit at which one oscillation can be discerned in the presence of the other, determined by uncertainties of $\sim 10^{\circ}$ in the fitted phases, is that the amplitude of the weaker oscillation should not be smaller than a factor of $\sim 5$ less than that of the stronger, i.e. dual oscillations can be discerned if $0.2 \leq k \leq 5$ (Provan et al., 2013). For $k$ outside this range only the properties of the stronger oscillation can be determined.

Results adapted from Figs. 6 and 7 of Provan et al. (2013) are shown in Fig. 4, spanning the same interval as Figs. 2 and 3. In Fig. 4a we show the rev-by-rev phase data relative to a guide phase of period $10.64 \mathrm{~h}$, where specifically we plot $\left(\Phi_{i}+\gamma_{i \mathrm{~N}}\right)-\Phi_{\mathrm{g}}=-\left(\psi_{i}-\gamma_{i \mathrm{~N}}\right)$ corresponding to "N format", such that the phases of each field component would fall on a common line for a pure northern oscillation. Red, green, and blue circles correspond to the $r, \theta$, and $\varphi$ field components, respectively, shown for clarity over two phase cycles. Figure $4 \mathrm{~b}$ similarly shows the same phase data relative to a guide phase of period $10.69 \mathrm{~h}$, where now we plot $\left(\Phi_{i}+\gamma_{i \mathrm{~S}}\right)-\Phi_{\mathrm{g}}=-\left(\psi_{i}-\gamma_{i \mathrm{~S}}\right)$ corresponding to "S format", such that the phases of each component would fall on a common line for a pure southern oscillation. When both oscillations are present, however, the beat-modulated phases are clustered about the northern phase in $\mathrm{N}$ format, and about the southern phase in $\mathrm{S}$ format, allowing both to be determined when $k$ lies within the above limits. The black lines show these phases determined using directional statistics methods, where in E1 we show the results of a running determination taking 25 data points at a time typically spanning $\sim 200$ days at steps of $\sim 10$ days (Andrews et al., 2012), while in E2E4 we show the results of single linear fits similarly spanning $\sim 200$ days between each abrupt transition (Provan et al., 2013). The northern and southern periods follow from the slopes of these lines, as shown by the blue and red lines for the northern and southern oscillations in panel (d) (and previously for the intervals concerned in Figs. 1 and 2). The beat modulation of the data about these phases allows subsequent determination of amplitude ratio $k$ shown by the black lines in panel (e), where the centre dotted line indicates $k=1$ (equal amplitudes), with $k$ being plotted below the line $(0 \leq k \leq 1)$, and $(1 / k)$ above $(1 \geq(1 / k) \geq 0)$. The modelled beat-modulated phases are shown in panels (a) and (b) by the green lines for the $\theta$ component and the purple lines for $(r, \varphi)$. Alternatively, and equivalently, $k$ may be determined from fits to the beat-modulated difference between the $\theta$ and $(r, \varphi)$ component phases shown in panel (c), where the red circles show $\Delta \Phi_{\theta-r}=\Phi_{\theta}-\Phi_{r}=\psi_{r}-\psi_{\theta}$ and blue circles $\Phi_{\theta}-\left(\Phi_{\varphi}+90^{\circ}\right)=\left(\psi_{\varphi}-90^{\circ}\right)-\psi_{\theta}$. The squares in E1 also show the results of an alternative fit methodology using simultaneous five-parameter fits to the phase data (two linear phases and $k$ ) employing 150-day segments of data separated by 50 days, as discussed in Sect. 2 in relation to Figs. 2 and 3, which show closely similar results (Provan et 


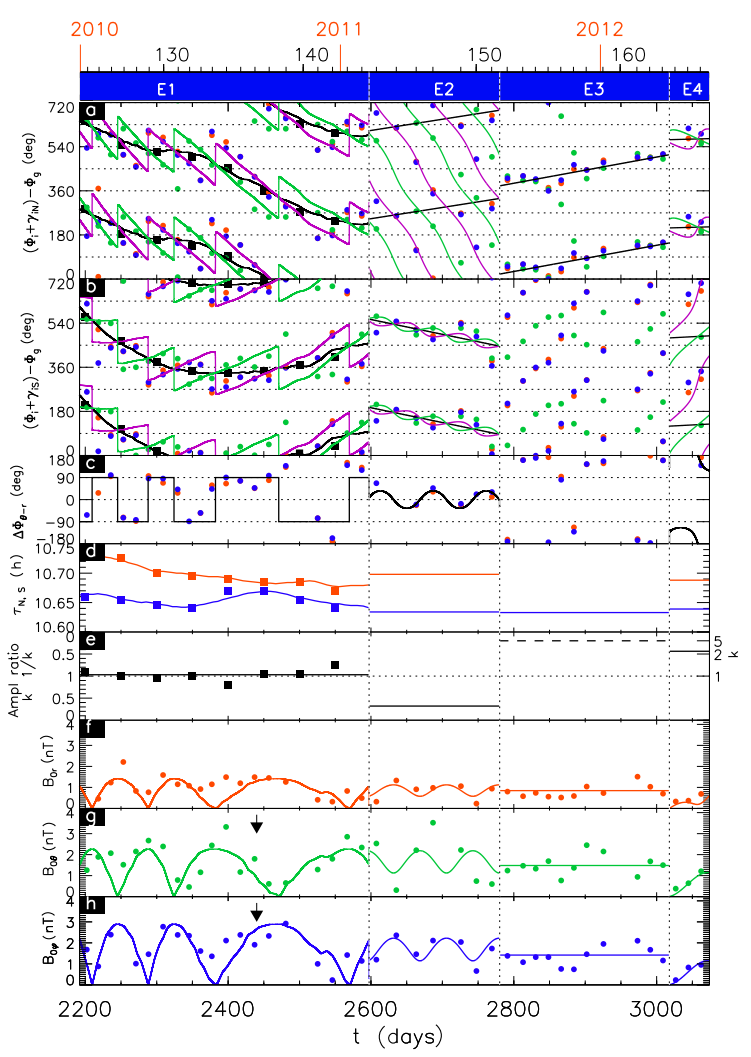

Figure 4. Summary plot of magnetic field oscillation data over the same interval as shown in Figs. 2 and 3, from the start of 2010 to mid-2012. From top to bottom we show (a) oscillation phase data in $\mathrm{N}$ format (see text) relative to a guide phase of $10.64 \mathrm{~h}$, where red, green, and blue data correspond to $r, \theta$, and $\varphi$ field components, shown for clarity over two phase cycles with all data plotted twice, (b) the same phase data in $\mathrm{S}$ format (see text) relative to a guide phase of $10.69 \mathrm{~h},(\mathbf{c})$ the phase difference between the $\theta$ and $(r, \varphi)$ field components $\Delta \Phi_{\theta-r}$ (Eq. 6), red corresponding to $\Phi_{\theta}-\Phi_{r}$ and blue to $\Phi_{\theta}-\left(\Phi_{\varphi}+90^{\circ}\right)$, (d) the oscillation periods, blue for the northern period and red for the southern, (e) the oscillation amplitude ratio north/south $k$, where the centre dotted line indicates $k=1$, with $k$ plotted directly below the line $(0 \leq k \leq 1)$, and $(1 / k)$ above $(1 \geq(1 / k) \geq 0)$, and (f)-(h) the amplitudes of the $r, \theta$, and $\varphi$ field components. The black lines in (a) and (b) show the northern and southern phases, respectively, determined from fits to the N- and S-format phase data (Andrews et al., 2012), while in E2-E4 we show the results of single linear fits between each abrupt PPO transition (Provan et al., 2013). The green ( $\theta$ component) and purple ( $r$ and $\varphi$ components) lines in (a) and (b) and the black lines in (c) show the model beat-modulated phase data derived using the northern and southern phases and $k$ values (using the central value $k=1$ in E1). Similarly, the coloured lines in (f)-(h) show the model beat-modulated oscillation amplitudes for the three field components. The squares in (a), (b), (d), and (e) in E1 show the results of simultaneous five-parameter fits to the phase data (Provan et al., 2013). Year boundaries, rev numbers, and interval identifiers are shown at the top of the plot as in Figs. 2 and 3, together with vertical dotted lines as also in Figs. 2 and 3. al., 2013). Figure $4 \mathrm{f}-\mathrm{h}$ then show the oscillation amplitude data for the three field components, together with the beatmodulated model fitted to the data using the northern and southern phases and the amplitude ratio $k$.

These results clearly show that $k \approx 1$ conditions (nearequal amplitudes) pertain throughout $\mathrm{E} 1$, with indications of slight southern dominance prior to the near-coalescence of the periods near $t=2450$ days (mid-September 2010) and slight northern dominance thereafter. Here the central value $k=1$ has been used to derive the beat-modulated model values in E1 in Fig. 4a-c and $\mathrm{f}-\mathrm{h}$. Provan et al. (2013) then find $k \approx 0.32$ in E2 (a return to southern dominance), $k>5$ in E3 (strongly northern dominant with no evident southern oscillation), and $k \approx 1.56$ in $\mathrm{E} 4$ (northern dominant but not as strongly as in E3), as indicated in Sect. 2. These changes are entirely evident in the varying beat modulations of the phase data shown in Fig. $4 \mathrm{a}$ and $\mathrm{b}$, with the phases in E1 rastering near-equally between $-90^{\circ}$ and $+90^{\circ}$ about the northern and southern phases in $\mathrm{N}$ and $\mathrm{S}$ format, respectively, while in E2 the phases are much more closely clustered in S format than in $\mathrm{N}$ format, and vice-versa in $\mathrm{E} 3$ with no evident clustering in $\mathrm{S}$ format in this case. Correspondingly, $\Delta \Phi_{\theta-r}$ in Fig. $4 \mathrm{c}$ varies about $0^{\circ}$ (the southern value) in E2 and about $\pm 180^{\circ}$ (the northern value) in E3, while switching between $-90^{\circ}$ and $+90^{\circ}$ in the two halves of the beat cycle in $\mathrm{E} 1$, this being characteristic of near-equal amplitudes. Similarly, the model beat-modulated amplitudes in Fig. $4 \mathrm{f}-\mathrm{h}$ show good overall agreement with the amplitude data, in particular showing antiphase variations in the amplitudes of the $\theta$ and $(r, \varphi)$ field components with the $\theta$ amplitude decreasing and the $(r, \varphi)$ amplitude increasing during the half-beat interval when $\Delta \Phi_{\theta-r}=+90^{\circ}$, and vice versa when $\Delta \Phi_{\theta-r}=-90^{\circ}$, this being the unequivocal signature of the beat phase given by Eq. (4) increasing with time, i.e. the northern period being shorter than the southern (Provan et al., 2013).

We note in passing, however, one brief interval in E1 where the amplitude data do not behave in the manner just described. If we examine the $\theta$ and $\varphi$ component amplitude data in Fig. $4 \mathrm{~g}$ and $\mathrm{h}$, which have the largest values and are less affected by measurement fluctuations than the $r$ component, we note that the data near $t \approx 2440$ days (rev 137 in early September 2010), marked by the vertical arrows, behave in the opposite sense to that expected above. That is, during an interval when $\Delta \Phi_{\theta-r} \approx+90^{\circ}$, the $\theta$ component amplitude briefly increased with time while the $\varphi$ component amplitude simultaneously decreased, opposite to the behaviour of the surrounding data. This represents evidence that the beat-phase $\Delta \Phi_{B}$ decreased with time during the previous $\sim 20$-day interval, implying that the two periods briefly crossed, northern longer than southern, and then crossed back again. If so, this behaviour is not quite captured by either of the magnetic period analyses shown, which use either $\sim 200$ day (Andrews et al., 2012) or 150-day (Provan et al., 2013) segments of phase data. Instead, these show the two periods as almost coalescing at a closely adjacent time as noted 
above. Although clearly tentative, this behaviour represents the only evidence in the magnetic field data for "crossed" periods during the interval shown (or indeed to date). Otherwise, the combined phase and amplitude data require the southern period to remain longer than the northern throughout.

Overall, these results are clearly inconsistent with those derived in interval E1 from SKR data by Fischer et al. (2014) as shown in Fig. 2, particularly the crossed periods, northern longer than southern, at the beginning of the interval shown, from mid-January to late March 2010. They are also inconsistent with the Fischer et al. (2014) interpretation of the SKR data as showing a phase-locked single period between early April 2010 and mid-February 2011 that we have shown in Sect. 2 segues from the magnetic southern period early in this interval into the magnetic northern period later in the interval, approximately in line with the modestly dominant oscillations then observed in the equatorial core magnetosphere. Instead, the magnetic field data demonstrate the presence of two oscillations with separated periods, with the southern longer than northern (with the possible brief exception mentioned above), and with near-equal amplitudes throughout E1. The key features are (a) near-equal clustering of the phase data about the northern and southern phases in $\mathrm{N}$ and $\mathrm{S}$ formats, with component phases that raster nearlinearly between $-90^{\circ}$ and $+90^{\circ}$ about these phases at the beat period, (b) altered relative phases between the oscillations in the $\theta$ and $(r, \varphi)$ field components, with $\Delta \Phi_{\theta-r}$ varying between $-90^{\circ}$ and $+90^{\circ}$ in the two halves of the beat cycle rather than around $180^{\circ}$ for northern-dominant oscillations and $0^{\circ}$ for southern-dominant oscillations, and (c) corresponding antiphase modulations of the $\theta$ and $(r, \varphi)$ component amplitudes. We again suggest that the reported apparent phase-locking of the SKR modulations in this interval results instead from the phenomenon reported by Lamy (2011) in which modulations associated with one hemisphere can appear in the polarization-separated data associated with the other. Indeed, Fischer et al. (2014) themselves report such behaviour in their data for interval E2 when two separate periods are again recognized in the SKR data (see their Fig. 5 and related discussion), with magnetically dominant "southern" signals being evident in "northern" (i.e. RH polarized) emissions.

\section{Relation of magnetic field oscillations with SKR data in interval E3}

The results in Fig. 4 thus provide unequivocal evidence in the magnetic field data for northern and southern PPO oscillations of separate periods essentially throughout interval E1 (and in interval E4 from the extended data set), contrary to the single phase-locked periods deduced from SKR data by Fischer et al. (2014). However, the same arguments do not hold for E3, a 240-day interval from mid-August 2011

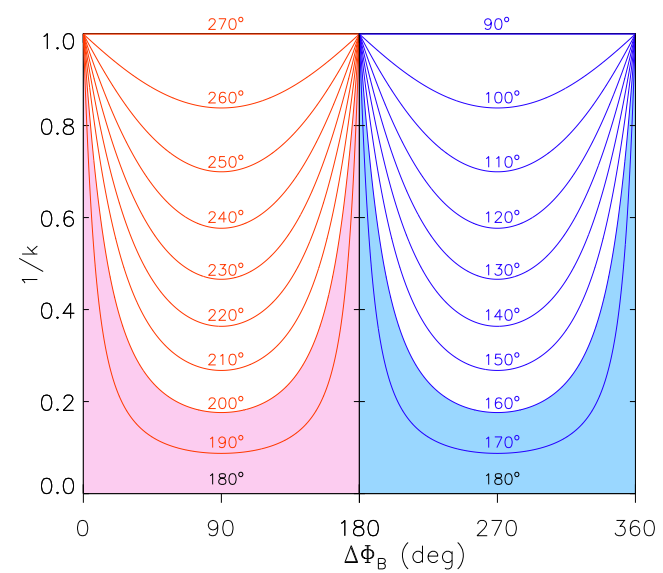

Figure 5. Contour plot of the phase difference between the $\theta$ and $(r$, $\varphi)$ field components $\Delta \Phi_{\theta-r}$, corresponding to $\Phi_{\theta}-\Phi_{r}$ or equivalently $\Phi_{\theta}-\left(\Phi_{\varphi}+90^{\circ}\right)$ (Eq. 6), plotted vs. $(1 / k)$ on the vertical axis over the range $0 \leq(1 / k) \leq 1$ (for northern-dominant oscillations), and vs. beat-phase $\Delta \Phi_{B}$ on the horizontal axis over the range $0^{\circ} \leq \Delta \Phi_{B} \leq 360^{\circ}$ (modulo $360^{\circ}$ ). Contours are shown at $10^{\circ}$ intervals between 270 and $180^{\circ}$ on the left (red), and between 180 and $90^{\circ}$ on the right.

to early April 2012 for which Fischer et al. (2014) again deduce phase-locked northern and southern modulations in SKR data (Figs. 2 and 3), but for which the magnetic phase data provide evidence only for oscillations with northern polarization, with no detectable clustering in S format (Fig. 4). Instead, the $\mathrm{N}$-format data are tightly clustered about the northern phase, with the exception of two adjacent phase data sets mid-interval that show greater scatter indicative of shortterm phase variability that is found to occur from time to time (e.g. the S-format data in E2). With the exception of these points, the $\Delta \Phi_{\theta-r}$ phase difference data in Fig. $4 \mathrm{c}$ show variations of $\sim \pm 20^{\circ}$ about a mean value of $\sim 170^{\circ}$, these being essentially at the noise level given a typical uncertainty in the individual phase determinations of $\sim 10^{\circ}$. This implies that any second oscillation producing beat modulations in the phase data must be smaller in amplitude than the northern oscillations by a factor of at least $\sim 5$, i.e. that $(1 / k) \leq 0.2$ (Provan et al., 2013).

As noted by Provan et al. (2014), however, this does not immediately preclude the presence of a southern oscillation of larger relative amplitude, provided that it remains either closely in phase or antiphase with the northern oscillations throughout E3, that is to say, a nearly phase-locked oscillation of nearly the same period as the northern oscillation, as suggested by the results of Fischer et al. (2014). In order to examine the requirements more closely, in Fig. 5 we show a contour plot of $\Delta \Phi_{\theta-r}$, plotted against $(1 / k)$ over the range $0 \leq(1 / k) \leq 1$ (for northern-dominant oscillations) on the vertical axis and beat-phase $\Delta \Phi_{B}$ over the full range $0^{\circ} \leq \Delta \Phi_{B} \leq 360^{\circ}$ on the horizontal axis. It can be shown from Eq. (3) that this phase difference is given by 
$\Delta \Phi_{\theta-r}=-\tan ^{-1}\left(\frac{2(1 / k) \sin \Delta \Phi_{B}}{(1 / k)^{2}-1}\right)$,

(see, for example, Provan et al. (2013), their Eq. 2g). In this expression the signs of the numerator and denominator are considered separately to define the arctangent function over the full $360^{\circ}$ range. The value of $\Delta \Phi_{\theta-r}$ is exactly $180^{\circ}$ on the bottom and vertical black bounding lines of the figure as indicated, i.e. the polarization is that of a pure northern oscillation if either $(1 / k)=0$ corresponding to no southern oscillation, or if $\Delta \Phi_{B}=0^{\circ}$ or $180^{\circ}$ (modulo $360^{\circ}$ ) corresponding to a southern oscillation of lesser amplitude than the northern that is either exactly in phase or in antiphase with the northern oscillation. The red contours on the left of Fig. 5 for $0^{\circ} \leq \Delta \Phi_{B} \leq 180^{\circ}$ then show $\Delta \Phi_{\theta-r}$ increasing vertically with $(1 / k)$ at $10^{\circ}$ steps from $180^{\circ}$ at the bottom to $270^{\circ}$ (equivalent to $-90^{\circ}$ modulo $360^{\circ}$ ) at the top, while the blue contours on the right similarly show $\Delta \Phi_{\theta-r}$ decreasing vertically from $180^{\circ}$ at the bottom to $90^{\circ}$ at the top. Thus for small values of $(1 / k)$, i.e. strongly northern-dominant oscillations, $\Delta \Phi_{\theta-r}$ varies modestly about $180^{\circ}$ over the beat cycle $\left(\Delta \Phi_{B}\right.$ varying between $0^{\circ}$ and $\left.360^{\circ}\right)$, while when $(1 / k)$ approaches unity, i.e. near-equal amplitudes, $\Delta \Phi_{\theta-r}$ flips between $-90^{\circ}$ and $+90^{\circ}$ in the two halves of the beat cycle, as indicated in Sect. 3.

The shaded region in Fig. 5 shows where the value of $\left|\Delta \Phi_{\theta-r}\right|$ lies within $\pm 20^{\circ}$ of $180^{\circ}$, consistent with the majority of the E3 data in Fig. 4. If we then consider the presence of a secondary southern oscillation of a significantly different period to the northern, such that the interval contains at least one beat cycle, then $(1 / k)$ must be less than $\sim 0.2$ if $\Delta \Phi_{\theta-r}$ is to remain within this range, i.e. the southern oscillation must be less than the northern by a factor of at least $\sim 5$, as indicated above. For larger values of $(1 / k)$, restriction of $\left|\Delta \Phi_{\theta-r}\right|$ to this range requires the relative phase of the oscillations $\Delta \Phi_{B}$ to be restricted to increasingly narrow ranges about $\Delta \Phi_{B}=0^{\circ}$ or $180^{\circ}$ (modulo $360^{\circ}$ ) as $(1 / k)$ increases toward unity, as indicated by the shaded regions. The two periods must therefore be sufficiently close so that $\Delta \Phi_{B}$ does not move out of these ranges throughout $\mathrm{E} 3$, with the oscillations closely either in phase or antiphase, i.e. they must constitute essentially phase-locked single periods, as proposed by Fischer et al. (2014) (we note, however, that the magnetic oscillations would have to be in antiphase to produce SKR modulations that are in phase from the two hemispheres as observed, associated with upward field-aligned currents that are co-located in local time in the two hemispheres, rather than on opposite sides of the planet). The difference in period allowed is given approximately by

$\delta \tau \approx \frac{\tau_{\mathrm{N}}^{2}}{T}\left(\frac{1-(1 / k)^{2}}{2(1 / k)}\right) \frac{\delta\left(\Delta \Phi_{\theta-r}\right)}{360}$,

where $\tau_{\mathrm{N}}$ is the northern period in E3 $\sim 10.63 \mathrm{~h}, T$ is the duration of $\mathrm{E} 3 \sim 240$ days, and the deviation in $\Delta \Phi_{\theta-r}$ is expressed in degrees. If we suppose, for example, that the southern amplitude is half the northern, such that $(1 / k)=$ 0.5 , then for a maximum phase deviation of $\sim 20^{\circ}$ we require $\delta \tau \leq 8 \times 10^{-4} \mathrm{~h}$, i.e. less than $3 \mathrm{~s}$. We note that this limiting period difference is approximately 2 orders of magnitude smaller than those measured in adjacent intervals E2 and E4, where the differences are $\sim 3-4 \mathrm{~min}$, in addition to which the phase difference has to lie closely either in phase or antiphase. Thus very particular conditions are required to have been maintained throughout this interval in this scenario.

The presence of such a southern oscillation will not only affect the phases, however, but also the relative amplitudes of the component oscillations. As shown by Eq. (3), if $\Delta \Phi_{B} \approx$ $0^{\circ}$ (modulo $360^{\circ}$ ) the southern oscillation will add to the northern for the $r$ and $\varphi$ components, but will subtract for the $\theta$ component, and vice versa if $\Delta \Phi_{B} \approx 180^{\circ}$. The presence of such a southern oscillation at significant amplitude relative to the northern will thus result in unusual values of the component amplitude ratios $R_{r / \theta}=\left(B_{0 r} / B_{0 \theta}\right)$ and $R_{\varphi / \theta}=\left(B_{0 \varphi} / B_{0 \theta}\right)$, both becoming larger than typical for $\Delta \Phi_{B} \approx 0^{\circ}$, and smaller than typical for $\Delta \Phi_{B} \approx 180^{\circ}$. The modified ratios are given by

$R_{(r, \varphi) / \theta}^{\prime}=\left(\frac{1 \pm(1 / k)}{1 \mp(1 / k)}\right) R_{(r, \varphi) / \theta}$,

where $R_{(r, \varphi) / \theta}$ represents the amplitude ratios for the individual northern and southern oscillations, $R_{(r, \varphi) / \theta}^{\prime}$ represents the corresponding ratios for the combined oscillations given by Eq. (3), and the upper signs correspond to $\Delta \Phi_{B} \approx 0^{\circ}$ and the lower to $\Delta \Phi_{B} \approx 180^{\circ}$. Thus, for example, if $(1 / k)$ has the significantly large value of $\sim 0.5$, say, we find $R^{\prime} \approx 3 R$ for $\Delta \Phi_{B} \approx 0^{\circ}$, while correspondingly $R^{\prime} \approx R / 3$ for $\Delta \Phi_{B} \approx$ $180^{\circ}$, effects that should be readily discernible in the amplitude data.

It is already evident from Fig. $4 \mathrm{f}$ and $\mathrm{h}$, however, that no large effects of this nature are present in E3; the amplitudes both in absolute and relative terms are comparable to those in adjacent intervals (with the exception of interval E4 where all amplitudes are severely reduced over an initial interval of $\sim 100$ days, Provan et al., 2013). More quantitatively, the ratios of the amplitudes found for earlier intervals $\mathrm{A}$ and $\mathrm{C}$ by Andrews et al. (2012) (see Fig. 1), together with those for adjacent intervals E1-E4 by Provan et al. (2013), excluding E3, are $R_{r / \theta}=0.65 \pm 0.19$ and $R_{\varphi / \theta}=1.22 \pm 0.29$. The values for E3 are $R_{r / \theta}^{\prime}=0.56$ and $R_{\varphi / \theta}^{\prime}=0.96$, both thus lying within the usual ranges. Noting, however, that both are slightly smaller than the overall mean values, we can use Eq. (7b) for $\Delta \Phi_{B} \approx 180^{\circ}$ (consistent with in-phase SKR emissions) to find the value of $(1 / k)=\left(R-R^{\prime}\right) /\left(R+R^{\prime}\right)$ that would account for these small deviations. From the ratio of $r$ and $\theta$ we find $(1 / k) \approx 0.06$, while from the ratio of $\varphi$ and $\theta$ we find $(1 / k) \approx 0.12$. This analysis thus shows that any such phase-locked southern oscillation must be much smaller in amplitude than that of the primary northern oscillation, being restricted to values $\sim 5-10 \%$ of the latter. We 
thus again conclude it to be likely that the phase-locked oscillations observed by Fischer et al. (2014) in the RH-polarized SKR emissions in E3 are due to the dual modulation phenomenon discussed above.

\section{Summary and conclusions}

In this paper we have discussed the divergent results on the nature of the PPOs in Saturn's magnetosphere in the postequinox interval presented from analysis of magnetic field oscillations by Andrews et al. (2012) and Provan et al. (2013, 2014), and from analysis of SKR modulations by Fischer et al. (2014). The comments on this topic presented subsequently by Fischer et al. (2015) will be the subject of separate discussion in due course. As shown by Andrews et al. (2012), the SKR and magnetic data sets are in good agreement in the pre-equinox southern summer interval showing well-separated periods of $\sim 10.6 \mathrm{~h}$ for the northern oscillations and $\sim 10.8 \mathrm{~h}$ for the southern, then converging towards a common period of $\sim 10.7 \mathrm{~h}$ over a $\sim 1$-year interval centred near equinox in mid-August 2009. In the postequinox interval, however, Fischer et al. (2014) report that the northern and southern SKR modulations, as determined from polarization-separated near-equatorial data, are characterized by phase-locked single periods over the interval from early 2010 to early 2013, with the exception of an interval from mid-February to late August 2011 when two separate periods were again observed, which they link to the occurrence of the GWS. By contrast, Andrews et al. (2012) and Provan et al. $(2013,2014)$ characterize this interval as being generally associated with continuing dual PPO periods that nearly coalesce in mid-September 2010, but then separate again with the northern period value of $\sim 10.64 \mathrm{~h}$ continuing shorter than the southern value of $\sim 10.69 \mathrm{~h}$. The magnetic oscillations are also shown to undergo abrupt (revto-rev) changes in relative amplitude at $\sim 100-200$-day intervals, as well as small changes in period and phase. The abrupt changes noted by Fischer et al. (2014) that occur after the beginning and near the end of the GWS event represent the first two of these transitions (defining interval E2), six of which have now been reported up until the end of 2013 as shown in Fig. 1 (Provan et al., 2014; Cowley and Provan, 2013).

Noting these differences in reported PPO properties, we make the statement of principle that the SKR and magnetic field phenomena cannot fundamentally show contrary properties, since both are believed to be directly related to the same underlying phenomenon, i.e. rotating magnetosphereionosphere coupling current systems that produce the magnetic field signatures directly via Ampère's law and the SKR emission signatures indirectly by acceleration and precipitation of electrons in upward-directed FAC sheets, leading to cyclotron maser unstable distributions. Indeed, we have shown that the Fischer et al. (2014) single phase-locked pe- riod corresponds to the southern magnetic period early in 2010, segues into the northern magnetic period in late 2010 (both in interval E1), and then returns back to the southern magnetic period again in mid-2012 (interval E4), in line, to a first approximation, with the variations in the dominant oscillation observed in the magnetic data. The exceptional interval in 2011 (E2), in which the southern modulation separates from the ongoing (northern magnetic period) modulations in the SKR data, leading to the re-emergence of dual periods, corresponds in the magnetic data to an interval of resumed southern dominance, in which the derived periods again agree well with the dual periods deduced from the SKR data. The jump in period by $\sim 0.05 \mathrm{~h}$ identified by Fischer et al. (2014) in this interval, which they relate to the GWS, is thus associated with the reappearance of ongoing southern oscillations in the SKR data related to the amplitude change in E2, and hence corresponds to the difference between the northern and southern periods rather than to an actual change in the PPO periods. The magnetic data do suggest an increase in southern period in this interval, but by a factor of less than half that suggested by Fischer et al. (2014) on the basis of their misidentification of the nature of the changes involved. The topic of the possible connection of these abrupt PPO changes with the GWS storm has previously been addressed by Cowley and Provan (2013), and need not be repeated here. We note, however, that while such changes certainly began during the GWS storm, many similar events have continued afterwards with $\sim 100-200$-day cadence, as illustrated in Fig. 1. Since the most recent of these events could clearly have no connection with the GWS, it seems reasonable to suppose that those occurring in February and August 2011 were similarly unconnected.

We have also reviewed the properties of the magnetic oscillations in the two intervals adjacent to E2 in which Fischer et al. (2014) report single phase-locked northern and southern SKR modulations. For the earlier interval in E1 spanning early April 2010 to mid-February 2011, we have shown that the magnetic data provide unequivocal evidence for the presence of northern and southern oscillations with near-equal amplitudes and separated periods throughout, the northern period remaining shorter than the southern (Provan et al., 2013, 2014). The evidence consists of clear variations of the oscillation phases and amplitudes at the beat period of the two oscillations, specifically phases that raster near-linearly through $\pm 90^{\circ}$ about the northern and southern phases, corresponding changes in the relative phases of the three field components, and antiphase modulations of the $\theta$ and $(r, \varphi)$ component amplitudes. The analysis also shows unequivocally that the northern magnetic period remains shorter than the southern throughout E1 (thus contradicting the opposite conclusions of Fischer et al. (2014) for the interval early in 2010 before apparent phase-locked SKR modulations appeared, see Fig. 2), though they converge closely in AugustSeptember 2010. Indeed, we have newly pointed out tentative evidence from the magnetic amplitude data that the two 
periods may briefly have crossed and re-crossed in late August to early September 2010. Though certainly tentative, this remains the only evidence for crossed periods, northern longer than southern, in the magnetic field data analysed to date (to late 2013). These results strongly suggest that the phase-locked single periods deduced by Fischer et al. (2014) are instead due to the phenomenon first reported by Lamy (2011) in which the period associated with one hemisphere is also found in the polarization-separated data associated with the other, particularly in near-equatorial data as is relevant here. The origin of this effect, whether physical or technique-associated, however, remains unclear.

Following interval E2, Fischer et al. (2014) again report the occurrence of phase-locked single northern and southern periods to mid-2012 (Fig. 2), corresponding to intervals E3 and the beginning of E4 of Provan et al. (2013, 2014). While separated northern and southern periods are again clearly present in the magnetic field data in E4, following a nearcomplete suppression of the oscillations early in that interval, only a single magnetic period is present in E3, the field component phases indicating a pure northern oscillation. In particular, no oscillations are evident near the ongoing southern periods before or after this interval (i.e. E2 and E4) within an upper limit of $\sim 20 \%$ of the northern amplitude. This finding, however, does not directly preclude the presence of a southern oscillation of larger relative amplitude provided, first, that its period is very close to that of the observed northern period such that the beat period is long compared with the $\sim 240$-day length of E3, and, second, that it is near to either in phase or antiphase with the northern oscillation, i.e. it is nearly phase-locked in this condition as suggested by Fischer et al. (2014). However, the presence of such an oscillation would then produce clear signatures in the relative amplitudes of the three spherical polar field components, since a southern oscillation would either have its $(r, \varphi)$ components in phase with the northern oscillation and its $\theta$ component in antiphase, or vice versa. No significant signatures of this nature are found in the amplitude data for this interval, however, showing that if such a southern oscillation is present, its amplitude is at most $\sim 5-10 \%$ of the dominant northern oscillation. We thus suggest it to be far more likely that the dual phase-locked oscillations found by Fischer et al. (2014) in E3 are again due to the presence of a single northern period of typical strength combined with the above SKR phenomenon discussed previously by Lamy (2011).

Acknowledgements. This work was supported by STFC consolidated grant ST/K001000/1.

The topical editor E. Roussos thanks the two anonymous referees for help in evaluating this paper.

\section{References}

Andrews, D. J., Bunce, E. J., Cowley, S. W. H., Dougherty, M. K., Provan, G., and Southwood D. J.: Planetary period oscillations in Saturn's magnetosphere: Phase relation of equatorial magnetic field oscillations and SKR modulation, J. Geophys. Res., 113, A09205, doi:10.1029/2007JA012937, 2008.

Andrews, D. J., Cowley, S. W. H., Dougherty, M. K., and Provan, G.: Magnetic field oscillations near the planetary period in Saturn's equatorial magnetosphere: Variation of amplitude and phase with radial distance and local time, J. Geophys. Res., 115 , A04212, doi:10.1029/2009JA014729, 2010a.

Andrews, D. J., Coates, A. J., Cowley, S. W. H., Dougherty, M. K., Lamy, L., Provan, G., and Zarka, P.: Magnetospheric period oscillations at Saturn: Comparison of equatorial and high-latitude magnetic field periods with north and south SKR periods, J. Geophys. Res., 115, A12252, doi:10.1029/2010JA015666, $2010 \mathrm{~b}$.

Andrews, D. J., Cowley, S. W. H., Dougherty, M. K., Lamy, L., Provan, G., and Southwood, D. J.: Planetary period oscillations in Saturn's magnetosphere: Evolution of magnetic oscillation properties from southern summer to post-equinox, J. Geophys. Res., 117, A04224, doi:10.1029/2011JA017444, 2012.

Badman, S. V., Andrews, D. J., Cowley, S. W. H., Lamy, L., Provan, G., Tao, C., Kasahara, S., Kimura, T., Fujimoto, M., Melin, H., Stallard, T., Brown, R.H., and Baines, K.H.: Rotational modulation and local time dependence of Saturn's infrared $\mathrm{H}_{3}^{+}$auroral intensity, J. Geophys. Res., 117, A09228, doi:10.1029/2012JA017990, 2012.

Burch, J. L., DeJong, A. D., Goldstein, J., and Young, D. T.: Periodicity in Saturn's magnetosphere: Plasma cam, Geophys. Res. Lett., 36, L14203, doi:10.1029/2009GL039043, 2009.

Carbary, J. F., Mitchell, D. G., Krimigis, S. M., and Krupp N.: Electron periodicities in Saturn's outer magnetosphere, J. Geophys. Res., 112, A03206, doi:10.1029/2006JA012077, 2007.

Cowley, S. W. H. and Provan, G.: Saturn's magnetospheric planetary period oscillations, neutral atmosphere circulation, and thunderstorm activity: Implications, or otherwise, for physical links, J. Geophys. Res., 118, 7246-7261, doi:10.1002/2013JA019200, 2013.

Fischer, G., Ye, S.-Y., Groene, J. B., Ingersoll, A. P., Sayanagi, K. M., Menietti, J. D., Kurth, W. S., and Gurnett, D. A.: A possible influence of the Great White Spot on Saturn kilometric radiation periodicity, Ann. Geophys., 32, 1463-1476, doi:10.5194/angeo32-1463-2014, 2014.

Fischer, G., Gurnett, D. A., Kurth, W. S., Ye, S.-Y., and Groene, J. B.: Saturn kilometric radiation periodicity after equinox, Icarus, 254, 72-91, 2015.

Galopeau, P. H. M. and Lecacheux A.: Variations of Saturn's radio rotation period measured at kilometer wavelengths, J. Geophys. Res., 105, 13089-13101, 2000.

Gurnett, D. A., Kurth, W. S., and Scarf, F. L.: Plasma waves near Saturn: Initial results from Voyager 1, Science, 212, 235-239, 1981.

Gurnett, D. A., Lecacheux, A., Kurth, W. S., Persoon, A. M., Groene, J. B., Lamy, L., Zarka, P., and Carbary, J. F.: Discovery of a north-south asymmetry in Saturn's radio rotation period, Geophys. Res. Lett., 36, L16102, doi:10.1029/2009GL039621, 2009.

Gurnett, D. A., Groene, J. B., Averkamp, T. F., Kurth, W. S., Ye, S.Y., and Fischer, G.: The SLS4 longitude system based on a track- 
ing filter analysis of the rotational modulation of Saturn kilometric radiation, in: Planetary Radio Emissions VII, edited by: Rucker, H. O., Kurth, W. S., Louarn, P., and Fischer, G., 51-64, Austrian Acad. Sci. Press, Vienna, 2011.

Hunt, G. J., Cowley, S. W. H., Provan, G., Bunce, E. J., Alexeev, I. I., Belenkaya, E. S., Kalegaev, V. V., Dougherty, M. K., and Coates, A. J.: Field-aligned currents in Saturn's southern nightside magnetosphere: Sub-corotation and planetary period oscillation components, J. Geophys. Res., 119, 9847-9899, doi:10.1002/2014JA020506, 2014.

Jia, X., Kivelson, M. G., and Gombosi, T. I.: Driving Saturn's magnetospheric periodicities from the upper atmosphere/ionosphere, J. Geophys. Res., 117, A04215, doi:10.1029/2011JA017367, 2012.

Kimura, T., Lamy, L., Tao, C., Badman, S. V., Kasahara, S., Cecconi, B., Zarka, P., Morioka, A., Miyoshi, Y., Maruno, D., Kasaba, Y., and Fujimoto, M.: Long-term modulations of Saturn's auroral radio emissions by the solar wind and seasonal variations controlled by the solar ultraviolet flux, J. Geophys. Res., 118, 7019-7035, doi:10.1002/2013JA018833, 2013.

Kurth, W. S., Averkamp, T. F., Gurnett, D. A., Groene, J. B., and Lecacheux, A.: An update to a Saturnian longitude system based on kilometric radio emissions, J. Geophys. Res., 113, A05222, doi:10.1029/2007JA012861, 2008.

Lamy, L.: Variability of southern and northern SKR periodicities, in: Planetary Radio Emissions VII, edited by: Rucker, H. O., Kurth, W. S., Louarn, P., and Fischer, G., 39-50, Austrian Acad. Sci. Press, Vienna, 2011.

Lamy, L., Zarka, P., Cecconi, B., Prangé, R., Kurth, W. S., and Gurnett, D. A.: Saturn kilometric radiation: Average and statistical properties, J. Geophys. Res., 113, A07201, doi:10.1029/2007JA012900, 2008a.

Lamy, L., Zarka, P., Cecconi, B., Hesse, S., and Prangé, R.: Modeling of Saturn kilometric radiation arcs and equatorial shadow zone, J. Geophys. Res., 113, A10213, doi:10.1029/2008JA013464, 2008b.

Lamy, L., Schippers, P., Zarka, P., Cecconi, B., Arridge, C. S., Dougherty, M. K., Louarn, P., André, N., Kurth, W. S., Mutel, R. L., Gurnett, D. A., and Coates, A. J.: Properties of Saturn kilometric radiation measured within its source region, Geophys. Res. Lett., 37, L12104, doi:10.1029/2010GL043415, 2010.

Lamy, L., Cecconi, B., Zarka, P., Canu, P., Schippers, P., Kurth, W. S., Mutel, R. L., Gurnett, D. A., Menietti, D., and Louarn P.: Emission and propagation of Saturn kilometric radiation: Magnetoionic modes, beaming pattern, and polarization state, J. Geophys. Res., 116, A04212, doi:10.1029/2010JA016195, 2011.
Nichols, J. D., Clarke, J. T., Cowley, S. W. H., Duval, J., Farmer, A. J., Gérard, J.-C., Grodent, D., and Wannawichian, S.: Oscillation of Saturn's southern auroral oval, J. Geophys. Res., 113, A11205, doi:10.1029/2008JA013444, 2008.

Nichols, J. D., Cecconi, B., Clarke, J. T., Cowley, S. W. H., Gérard, J.-C., Grocott, A., Grodent, D., Lamy, L., and Zarka, P.: Variation of Saturn's UV aurora with SKR phase, Geophys. Res. Lett., 37, L15102, doi:10.1029/2010GL044057, 2010a.

Nichols, J. D., Cowley, S. W. H., and Lamy, L.: Dawn-dusk oscillation of Saturn's conjugate auroral ovals, Geophys. Res. Lett., 37, L24102, doi:10.1029/2010GL045818, 2010b.

Provan, G., Cowley, S. W. H., Sandhu, J., Andrews, D. J., and Dougherty, M. K.: Planetary period magnetic field oscillations in Saturn's magnetosphere: Post-equinox abrupt non-monotonic transitions to northern system dominance, J. Geophys. Res., 118, 3243-3264, doi:10.1002/jgra.50186, 2013.

Provan, G., Lamy, L., Cowley, S. W. H., and Dougherty, M. K.: Planetary period oscillations in Saturn's magnetosphere: Comparison of magnetic oscillations and SKR modulations in the post-equinox interval, J. Geophys. Res., 119, 7380-7401, doi:10.1002/2014JA020011, 2014.

Smith, C. G. A.: A Saturnian cam current system driven by asymmetric thermospheric heating, Mon. Not. R. Astron. Soc., 410, 2315-2329, doi:10.1111/j.1365-2966.2010.17602.x, 2010.

Southwood, D. J. and Cowley, S. W. H.: The origin of Saturn magnetic periodicities: Northern and southern current systems, J. Geophys. Res., 119, 1563-1571, doi:10.1002/2013JA019632, 2014.

Southwood, D. J. and Kivelson, M. G.: Saturn magnetospheric dynamics: Elucidation of a camshaft model, J. Geophys. Res., 112, A12222, doi:10.1029/2007JA012254, 2007.

Southwood, D. J. and Kivelson, M. G.: The source of Saturn's periodic radio emissions, J. Geophys. Res., 114, A09201, doi:10.1029/2008JA013800, 2009.

Warwick, J. W., Pearce, J. B., Evans, D. S., Carr, T. D., Schauble, J. J., Alexander, J. K., Kaiser, M. L., Desch, M. D., Pedersen, B. M., Lecacheux, A., Daigne, G., Boischot, A., and Barrow, C. H.: Planetary radio astronomy observations from Voyager-1 near Saturn, Science, 212, 239-243, 1981.

Warwick, J. W., Evans, D. S., Romig, J. H., Alexander, J. K., Desch, M. D., Kaiser, M. L., Aubier, M., Leblanc, Y., Lecacheux, A., and Pedersen, B. M.: Planetary radio astronomy observations from Voyager-2 near Saturn, Science, 215, 582-587, 1982.

Zarka, P.: Auroral radio emissions at the outer planets: Observations and theories, J. Geophys. Res., 103, 20159-20194, 1998. 